\title{
Improved Synchronization of HeartLander Locomotion with Physiological Cycles
}

\author{
Diego Moral del Agua, Nathan A. Wood, and Cameron N. Riviere \\ The Robotics Institute, Carnegie Mellon University, Pittsburgh, PA 15213 USA
}

\begin{abstract}
The variation of intrapericardial pressure due to the respiration and cardiac cycles greatly affects the locomotion efficiency of HeartLander, a minimally invasive surgical robot which adheres to and moves over the surface of the heart. This paper presents work which would allow HeartLander to improve motion efficiency by synchronizing its movement with the physiological cycles using a magnetic tracker. Using models of the respiratory and cardiac cycles generated using Kalman filtering techniques the work presented determines the phase of each physiological cycle in real time, which in turn enables synchronization of HeartLander to maximize locomotion efficiency.
\end{abstract}

\section{INTRODUCTION}

HeartLander, shown in Fig. 1, is a miniature mobile robot that crawls over the surface of the heart in order to provide therapy in a minimally invasive manner. HeartLander has demonstrated successful locomotion and precise target acquisition on the surface of the porcine beating heart in vivo, and multiple possible therapies have been proposed using this platform [1].

HeartLander moves in an inchworm-like fashion, alternately extending its front foot and drawing up ("retracting") its back foot using drive wire actuation and suction to adhere to the surface of the heart. Locomotion occurs in the intrapericardial space where the pressure applied to HeartLander by the pericardium varies significantly during the cardiac and respiratory cycles. In order for HeartLander to move efficiently, its locomotion has been timed to coincide with minimum intrapericardial pressure.

The phase value notation of Shechter et al. is used to describe the physiological cycles [2]. Using this notation the respiratory cycle is parameterized with expiration occurring in the range -1 to 0 and inspiration occurring in the range from 0 to 1 . The cardiac cycle is parameterized with systole

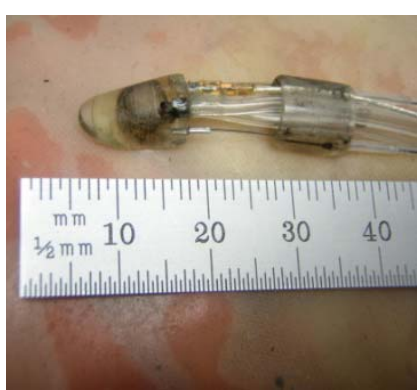

Figure 1. HeartLander. occurring in the range from 0 to 0.42 and diastole occurring in the range from 0.42 to 1 . Minimum lung volume occurs at the end of expiration, a phase value of 0 , and minimum heart volume occurs at the end of systole, a phase value of 0.42 .

Synchronization of HeartLander locomotion using

This work was supported by NIH grant no. R01 HL078839. accelerometry has been reported previously [3]. However, for simplicity of operation, the present work describes an improved method for synchronization using 6-DOF position feedback from the magnetic tracker that is already incorporated within the robot. Separated cardiac and respiratory motion estimation models are generated using Kalman filtering techniques. Using these models it is possible to determine the phase values of the cardiac and respiratory cycles. The phase values can then be used to ensure locomotion occurs only when intrapericardial pressure is low and motion efficiency will be maximized.

\section{METHODS}

\section{A. Motion Simulation}

Cardiac and respiratory motions are simulated in the laboratory using a beating heart phantom (Model 1283, The Chamberlain Group, Great Barrington, Mass.) mounted on an actuated linear slide. The heart model simulates the deformation of the surface of the heart during the cardiac cycle, while the linear slide simulates respiratory motion. Each stage of the motion phantom allows for control of frequency while the breathing stage also allows control of amplitude.

\section{B. Motion Model}

A model of the motion of the heart which considers the separate components, cardiac and respiratory, as Fourier series is estimated from the 6-DOF position measurements of HeartLander using an Extended Kalman filter as in [4]. The filter recursively estimates the parameters of the Fourier series, $F$, which then can be used to reconstruct the cardiac, $C$, and respiratory, $R$, components of the heart motion, as shown in Fig. 2. The model of the motion is given as:

$$
\begin{gathered}
F=R+C+D C \\
R=\sum_{n=1}^{H_{r}}\left[a_{n} \sin \left(n \sum_{i=0}^{k} \omega_{r_{i}} \delta t_{i}\right)+b_{n} \cos \left(n \sum_{i=0}^{k} \omega_{r_{i}} \delta t_{i}\right)\right] \\
C=\sum_{n=1}^{H_{c}}\left[c_{n} \sin \left(n \sum_{i=0}^{k} \omega_{c_{i}} \delta t_{i}\right)+d_{n} \cos \left(n \sum_{i=0}^{k} \omega_{c_{i}} \delta t_{i}\right)\right]
\end{gathered}
$$

where $D C$ is a constant offset, $H_{r}$ and $H_{c}$ are the number of respiratory and cardiac harmonics, $\omega_{r}$ and $\omega_{c}$ are the respiratory and cardiac base frequencies, and $\delta t$ is the time step. The Fourier coefficients are $a$ and $b$ for respiratory motion, and $c$ and $d$ for cardiac motion. 


\section{Estimation of Phase}

In order to determine the current respiratory and cardiac phase of the system the phase shifts, $\phi_{n}$, are found.

$$
\begin{aligned}
& \phi_{r_{n}}=\operatorname{atan} 2\left(-a_{n}, b_{n}\right) \\
& \phi_{c_{n}}=\operatorname{atan} 2\left(-c_{n}, d_{n}\right)
\end{aligned}
$$

Using these phase shifts, the respiratory and cardiac phase values, $\theta_{r}$ and $\theta_{c}$, using the notation of [2], are approximated using the first order phase angle.

$$
\begin{aligned}
\theta_{r} & =\frac{\bmod \left(\sum_{i=0}^{k} \omega_{r_{i}} \delta t_{i}+\phi_{r_{1}}, 2 \pi\right)}{\pi}-1 \\
\theta_{c} & =\frac{\bmod \left(\sum_{i=0}^{k} \omega_{c_{i}} \delta t_{i}+\phi_{c_{1}}, 2 \pi\right)}{2 \pi}
\end{aligned}
$$

\section{RESULTS}

HeartLander was placed on the anterior surface of the heart model. The heart model was set to have a cardiac frequency of approximately $1 \mathrm{~Hz}$, while the respiratory motion was set to approximately $0.25 \mathrm{~Hz}$ with a peak-to-peak amplitude of 4 $\mathrm{mm}$ [2]. Position data were recorded from HeartLander for approximately $180 \mathrm{~s}$. The parameters of the Fourier series were then estimated using the Extended Kalman filter. The separated respiratory and cardiac motions constructed from these parameters are also shown in Fig. 2(b).

Using the methods described previously, the anatomical phases were then determined using the Fourier series parameters at each instant as shown in Fig. 2. The green horizontal lines in Fig. 2(c) and 2(d) represent the bounds of the acceptable phase range, in which HeartLander achieves maximum locomotion efficiency. These ranges were previously established as $[-0.75,0]$ for respiratory phase and $[0.17,0.67]$ for cardiac phase [3].

The motion of HeartLander was simulated over the physiological cycles using a $10 \mathrm{~mm}$ step length with a step duration of 0.25 seconds. The phase of each step event (the start and end of motion for each foot) was collected for 50 cycles, totaling 200 step events. Without synchronization the distributions of step events over physiological phase, shown in Fig. 3, are nearly uniform, as expected. With synchronization the distributions of step events, shown in Fig. 4, are concentrated largely between the acceptable bounds, as expected. Those events that fall outside of the bounds usually correspond to the end (not the start) of a step.

\section{DISCUSSION}

Using Fourier series models of the respiratory and cardiac cycles, generated in real time using Kalman filtering techniques, the phase value of each cycle is identified using the output of the magnetic tracker incorporated within HeartLander. Using this phase information, locomotion of HeartLander can be timed to occur when intrapericardial pressure is lowest, in order to maximize step efficiency.

Future work will include refinement of the Kalman filtering techniques, followed by animal testing in vivo.
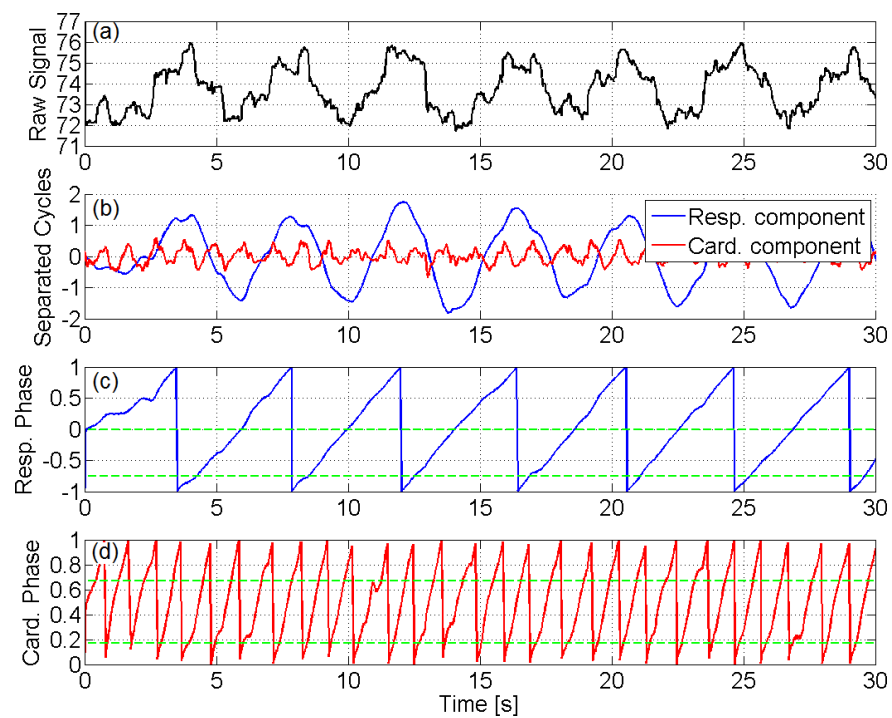

Fig. 1. (a) Measured signal of HeartLander motion on surface of phantom. (b) Separated respiratory and cardiac signals. (c) Respiratory Phase values with its synchronization interval $[-0.75,0]$. (d) Cardiac phase with its synchronization interval $[0.17,0.67]$.
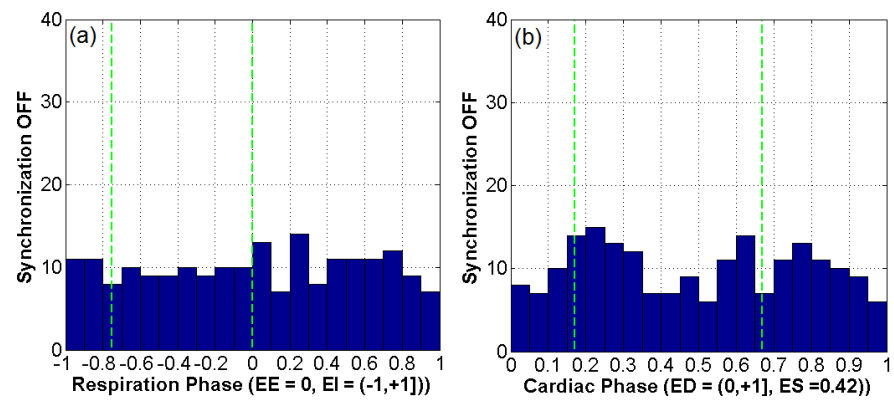

Fig. 2. (a) Respiration phase and (b) cardiac phase values of HeartLander motion when locomotion is unsynchronized.
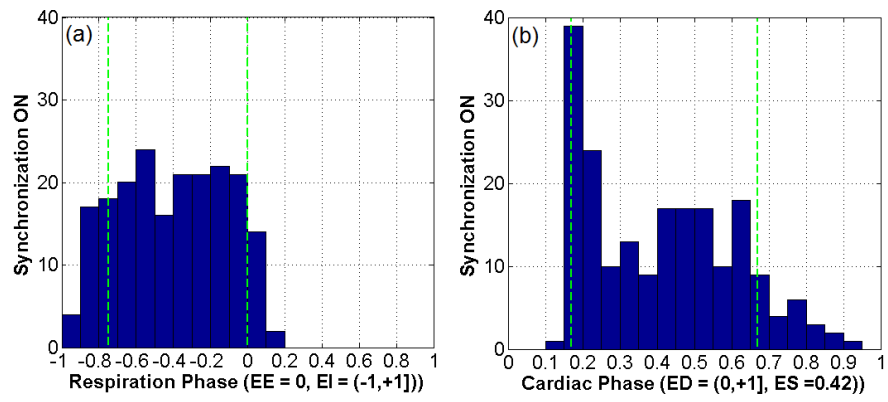

Fig. 4. (a) Respiration phase and (b) cardiac phase values of HeartLander motion when locomotion is synchronized.

\section{REFERENCES}

[1] N. A. Patronik, T. Ota, M. A. Zenati, and C. N. Riviere, "A miniature mobile robot for navigation and positioning on the beating heart," IEEE Trans. Rob., vol. 25, no. 5, pp. 1109-24, 2009

[2] G. Shechter, J. R. Resar, and E. R. McVeigh, "Displacement and velocity of the coronary arteries: cardiac and respiratory motion," IEEE Trans. Med. Imaging, vol. 25, no. 3, pp. 369-75, March 2006.

[3] N. A. Patronik, T. Ota, M. A. Zenati, and C. N. Riviere, "Synchronization of epicardial crawling robot with heartbeat and respiration for improved safety and efficiency of locomotion,” Int. J. Med. Robot. Comput. Assist. Surg., submitted.

[4] R. Richa, A. P. L. Bó, and P. Poignet, "Beating heart motion prediction for robust visual tracking,” Proc. IEEE Int. Conf. Rob. Autom., pp. 4579-84, 2010. 